\title{
Chronic pain after inflatable penile prosthesis implantation: An important complication to discuss with patients
}

\author{
David Chung, MD; Premal Patel, MD, FRCSC
}

Section of Urology, Department of Surgery, University of Manitoba, Winnipeg, MB, Canada

Cite as: Chung D, Patel P. Chronic pain after inflatable penile prosthesis implantation: An important complication to discuss with patients. Can Urol Assoc J 2022;16(2):47. http://dx.doi. org/10.5489/cuaj.7746

See related article on page 42

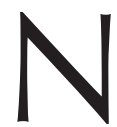
ew onset chronic pain following inflatable penile prosthesis (IPP) implantation is an important but poorly understood topic. This month's article by Campbell et al sought to shed further light on the matter by investigating the persistence of chronic pain following revision or explanation of the IPP.

Their case series identified 31 patients requiring surgical intervention (revision or explantation) for a new diagnosis of chronic penile prosthesis pain. Only 13 (42\%) patients reported improvement in pain after intervention. Of the patients who reported improvement of their pain, $84.6 \%$ initially localized their pain to their penis. ${ }^{1}$ Patients with persistent pain initially localized their pain to the pelvic and scrotal regions. In keeping with their hypothesis, all patients with pain secondary to device malposition reported pain resolution following intervention. ${ }^{1}$ The real possibility of developing chronic post-surgical pain (CPSP) following implantation of IPP makes it an important topic to counsel patients on when discussing the initial surgery. As shown in the study by Campbell et al, revision of the IPP is unlikely to be effective for treatment of de novo CPSP, especially if it is not associated with an anatomically correctable etiology.

CPSP is an unfortunate outcome that has a significant effect on a patient's morbidity. The currently accepted paradigm is that the development of CPSP is multifactorial, beginning from nerve injury and wound inflammatory response during the operation, thereafter, leading to peripheral and central sensitization. ${ }^{2}$ One retrospective study reported the prevalence of CPSP after urological surgery to be $24 \%,{ }^{3}$ a value comparable to other studies in the field.

Once a diagnosis of CPSP is suspected, it is important to properly identify the condition and any underlying etiology, while appropriately investigating for more sinister causes of pain in the postoperative period. The management strategy should be tailored to address the multifactorial nature of the problem, as well as involve counselling and education about the condition to the patient. Available pharmacother- apy agents include non-steroidal anti-inflammatory drugs, as well as medications aimed to treat the neuropathic element of CPSP, such as gabapentin, tricyclic antidepressants, and selective serotonin reuptake inhibitors. ${ }^{2}$ Other interventions include pelvic floor physiotherapy, cognitive behavioral therapy, and pain interventions in the form of regional blocks. However, as most of the literature regarding management of CPSP is extrapolated from other chronic pain disorders, further studies are required to gain better understanding of this pathology.

In terms of chronic pain following implantation of IPP, Campbell et al demonstrated that device malposition is a surgically correctable etiology that would benefit from revision/explantation. The presence of penile pain may be an important element of the history, possibly suggestive of a device-related etiology that can be corrected vs. pelvic/ scrotal pain that may be more suggestive of a chronic pain disorder. These patients would be ill-suited for a redo operation and should be managed akin to a CPSP patient.

The possibility of developing chronic pain following implantation of IPP is an important complication to discuss when obtaining consent from the patient. The nature of a potential chronic pain disorder should also be discussed, and that persistent pain may be experienced despite re-operation. Clinicians should also do their part to identify patients who are at high risk of developing CPSP. Potential surgical candidates with a history of chronic pain syndromes should be thoroughly counselled on the risk and implications of persistent pain following implantation of IPP that may be refractory to revision surgery.

Competing interests: The authors do not report any competing personal or financial interests related to this work.

\section{References}

1. Campbell JD, Chan EP, Pierdominico A Di, et al. Chronic pain associated with penile prostheses may persist despite revision or explantation. Can Urol Assoc J 2022;16:42-6. https://doi.org/10.5489/cuaj.7391

2. Visser EJ. Chronic post-surgical pain: Epidemiology and clinical implications for acute pain management. Acute Pain 2006;8:73-81. https://doi.org/10.1016/i.acpain.2006.05.002

3. Artus M, Laviolle B, Maurice A, et al. Risk factors for persistent pain after urological surgery. Ann Fr Anesth Reanim 2014;33:e89-94. https://doi.org/10.1016/i.annfar.2014.03.013

Correspondence: Dr. Premal Patel, Section of Urology, Department of Surgery, University of Manitoba, Winnipeg, MB, Canada; premalpatel8@gmail.com 